\title{
The companion of HD 190228: Planet or brown dwarf?^
}

\author{
Y. Q. Chen ${ }^{1,2}$ and G. Zhao ${ }^{1,2}$ \\ 1 Beijing Astronomical Observatory, Chinese Academy of Sciences, Beijing 100012, PR China \\ ${ }^{2}$ National Astronomical Observatories, Chinese Academy of Sciences, Beijing 100012, PR China
}

Received 27 March 2001 / Accepted 20 April 2001

\begin{abstract}
A detailed abundance analysis has been carried out from high-resolution, high signal-to-noise spectra of the G5IV star HD 190228, which is announced to harbor an extrasolar planet with $M_{\mathrm{P}} \sin i$ of $5.0 M_{\mathrm{J}}$ and an orbital period of 1127 days. Based on the model atmosphere of $T_{\text {eff }}=5180 \mathrm{~K}, \log g=3.7, \xi_{\mathrm{t}}=1.3 \mathrm{~km} \mathrm{~s}^{-1}$, we obtained $[\mathrm{Fe} / \mathrm{H}]=-0.40$, which puts it on the metal-poor tail of the metallicity distribution of the so far discovered 48 planet-harboring stars. The relative abundance, $[\mathrm{X} / \mathrm{Fe}]$, indicates an overabundance of light elements (O, Na, Mg, Al, Si, S, Sc) by 0.1-0.2 dex and the solar abundance of heavier elements (K, Ca, Ti, V, Cr, Mn, $\mathrm{Ni}, \mathrm{Ba})$. These elements show no conspicuous anomalies. The solar $[\mathrm{C} / \mathrm{Fe}]$ seems to be slightly smaller than the average (but within the scatter) of field stars of the same $[\mathrm{Fe} / \mathrm{H}]$, and there is no clear trend of $[\mathrm{X} / \mathrm{H}]$ with condensation temperature of the element. Neither the process of planet formation nor the stellar dilution during the subgiant stage seems to have polluted its chemical composition. The initially low metallicity may be explained by the suggestion that HD 190228 is accompanied by a brown dwarf instead of a planet.
\end{abstract}

Key words. stars: abundances - planetary system

\section{Introduction}

Recent radial-velocity surveys of solar-type stars have found many planetary candidates (http://www.obspm.fr), and many spectroscopic studies have been carried out in searching for the abundance anomalies of the parent stars. So far, the most significant result (e.g. Gonzalez et al. 2001) has been the high mean metallicity relative to the average of nearby solar-type stars $([\mathrm{Fe} / \mathrm{H}] \sim-0.2)$, and some scenarios (e.g. Gonzalez 1997) have been proposed to explain, physically, the link between the high metallicity and the formation of the planetary system. However, before such a link is used to constraint the planet formation theory, much attention should be paid to explain a few exceptions, i.e. stars on the metal-poor tail of the metallicity distribution of extrasolar planet host stars. On the other hand, a reasonable explanation of their relatively low metallicity is a potential support for the connection of the high metallicity with the presence of the planetary system.

Such low metallicity planet host stars are, actually, very few if we do not include those stars accompanied by brown dwarfs, which are beyond the scope of the planet formation theory. Among at least 40 planet host stars

Send offprint requests to: G. Zhao,

e-mail: zg@orion.bao.ac.cn

* Based on observation carried out at Beijing Astronomical Observatory (Xinglong, PR China). that have spectroscopic $[\mathrm{Fe} / \mathrm{H}]$ in the literature, there are only three cases, HD 6434, HD 37124 and HD 143761 with $[\mathrm{Fe} / \mathrm{H}]$ of $-0.54,-0.41$ and -0.29 , respectively. In this paper, we add another case, HD $190228([\mathrm{Fe} / \mathrm{H}]=-0.4)$, which has no any previous spectroscopic analysis. This star is also special in the characteristics of the companion. According to Marcy et al. (1999), the majority of planetary candidates have masses below $4 M_{\mathrm{J}}$ and there is a high occurrence of planets with semi-major axes smaller than 0.3 AU. The companion around HD 190228 is massive $\left(5.0 M_{\mathrm{J}}\right)$ and has long period (>1000 days) with an orbit beyond 2 AU. Finally, being a subgiant, the study of HD 190228 may provide some hints about the effect of stellar evolution on stellar surface composition and the existence of the planetary system.

\section{Observation}

The observation was performed on Dec. 8, 2000 using the coude echelle spectrograph attached to the $2.16 \mathrm{~m}$ telescope of Beijing Astronomical Observatory (Xinglong, China). The spectra were recorded by a Tek CCD detector of $1024 \times 1024$ pixels (with a size of $24 \mu \mathrm{m}$ ) and the spectral coverage was $550-820 \mathrm{~nm}$. The resolution is 37000 and the signal-to-noise is around 200.

The data reduction follows standard routines for order definition, background subtraction, wavelength calibration, radial velocity shift and continuum 
normalization. The equivalent widths $(E W \mathrm{~s})$ were measured by direct integration and Gaussian fitting.

\section{Analysis}

The effective temperature was estimated from Strömgren photometric indices (Olsen 1993) by using the calibration of Alonso et al. (1996). As seen in Thorén \& Feltzing (2000), the temperature estimated from Strömgren $(b-y)$ seems to be consistent with that derived from spectroscopic analysis for stars with $T_{\text {eff }} \gtrsim 5000 \mathrm{~K}$. No reddening correction was applied because $\mathrm{H} \beta$ is not available in the literature, but this may not be significant for a distance of $62 \mathrm{pc}$ and it was checked by the lack of the apparent trend of the derived iron abundance with excitation potential of low energy level. The surface gravity was calculated from Hipparcos parallax (ESA 1997) and the initial metallicity was estimated from the photometric $m_{1}$ based on Schuster \& Nissen (1989) calibration. The microturbulence, was obtained by requiring a zero slope of $[\mathrm{Fe} / \mathrm{H}]$ vs. $E W$. The whole procedure of deriving $T_{\text {eff }}, \log g$ and $\xi_{\mathrm{t}}$ was iterated to consistency when spectroscopic $[\mathrm{Fe} / \mathrm{H}]$ was obtained.

With the determined atmospheric parameters, we can derive the mass and age from theoretical evolutionary tracks and isochrones by a comparison of stellar position with the predicted HR diagram of VandenBerg et al. (2000). The kinematics are calculated from the Hipparcos parallax and radial velocity derived from the Doppler shift of the spectral lines. The calculations of galactic space velocity $(U, V, W)$ and orbital parameter $(R, Z)$ are the same as that presented in Johnson \& Soderblom (1987) and Allen et al. (1991).

The abundance analysis was based on a net of flux constant, homogeneous, LTE model atmospheres interpolated from the extensive grids of Kurucz (1993). With the code ABONTEST8, which is kindly provided by Pierre Magain, abundances were derived by requiring that the calculated equivalent width from the model should match the observed one. The mean abundance was derived from all available lines by giving equal weight to each line. Based on the solar abundances derived from moon spectra, stellar abundances were relative to the sun. The details for line selection, atomic line data, calculations of the uncertainties and the contribution from systematic errors are the same as those discussed in Chen et al. (2000). As expected, errors are around 0.05 dex for relative abundance, $[\mathrm{X} / \mathrm{Fe}]$, and of the order of 0.1 dex for $[\mathrm{X} / \mathrm{H}]$.

According to the non-LTE grids calculated by Takeda (private communication), this effect is negligible for S I $\lambda 869.3 \mathrm{~nm}$ and $\lambda 869.4 \mathrm{~nm}$ with a deviation less than 0.05 dex for both of this star and the sun. For the oxygen triplet, the difference between LTE and non-LTE value is around 0.1 dex, but it is similar for this star and the sun. Thus the non-LTE effect on the abundance relative to the sun is nearly canceled out.

Table 1 presents the stellar basic parameters, the companion information, kinematics and relative abundances.

\section{Results and discussions}

\subsection{Metallicity}

So far, the presence of planets and stellar metallicity are related by two possible scenarios: either the formation of the planetary system enhances its metallicity by the accretion of $\mathrm{H}$ and $\mathrm{He}$ depleted material, or the planet forms preferentially in more metal-rich environments via the initial condensation of "ice core" from metals. For HD 190228, the metallicity is -0.40 , which seems to be lower than those of the majority of planet host stars with a peak at $[\mathrm{Fe} / \mathrm{H}]=+0.1$. Thus, it is difficult to assume that the metallicity has been enhanced by the process of the planet formation. From the average age-metallicity and $R_{\mathrm{m}}-[\mathrm{Fe} / \mathrm{H}]$ relations of disk stars presented in Gonzalez (1999), it may have $[\mathrm{Fe} / \mathrm{H}]$ above -0.1 for its age of $\sim 3$ Gyr and +0.10 for its mean galactocentric distance in the stellar orbits, $R_{\mathrm{m}}=7.2 \mathrm{kpc}$.

We prefer the suggestion that the metallicity of this star could be unchanged since it was formed from the interstellar medium, because it is within the scatter of these relations. Furthermore, the potential existence of a vertical gradient (Cui et al. 2000) in the disk partly explains its low metallicity. Based on the $Z_{\max }$ of $0.37 \mathrm{kpc}$, the metallicity is around $-0.22 \pm 0.2$, which is much closer to the present value. The $V_{\mathrm{LSR}}$ of $-40 \mathrm{kms}^{-1}$ indicates a slight lag in the galactic rotation velocity relative to the solar neighbourhood. But it is unreasonable to relate the low metallicity and the lag of the galactic rotation with a thick-disk origin due to its young age.

\subsection{Abundances}

The abundance pattern of this star is quite normal for its metallicity. As shown in Fig. 1, the abundances relative to $\mathrm{H}$ are all under solar, and the relative abundances with respect to Fe show a noticeable enhancement of light elements (Na, Mg, Al, Si) by $\sim 0.15$ dex and $\mathrm{S}$ by 0.20 dex, while heavier elements ( $\mathrm{Ca}, \mathrm{Ti}, \mathrm{V}, \mathrm{Cr}, \mathrm{Mn}, \mathrm{Ni}, \mathrm{Ba}$ ) are nearly solar. The $[\mathrm{O} / \mathrm{Fe}]$ is 0.23 dex with a negligible nonLTE correction from Takeda's grids. The adopted value is 0.08 dex by using the scaled relation between the triplet $\lambda 777 \mathrm{~nm}$ and the forbidden [O I] $\lambda 630 \mathrm{~nm}$ presented in Edvardsson et al. (1993). Sc is 0.11 dex enhanced, while $\mathrm{K}$ is slightly underabundant. An interesting result is that the $[\mathrm{C} / \mathrm{Fe}]$ is solar. Although this is 0.12 dex lower than the average of the nearby solar-type stars at $[\mathrm{Fe} / \mathrm{H}]=-0.4$ (Gustafsson et al. 1999; Shi et al. 2001), the scatter of C abundance at this point is larger than 0.2 dex. Thus the solar $[\mathrm{C} / \mathrm{Fe}]$ is not a significant deviation from those of normal field stars.

Alternatively, we inspect to see if there is a signature of the polluted material by the process of the planet formation with which the slightly low $[\mathrm{C} / \mathrm{Fe}]$ and the enhanced $[\mathrm{X} / \mathrm{Fe}]$ of $\mathrm{Al}, \mathrm{Si}, \mathrm{Sc}$ might be associated. Theoretically, it has been suggested (Lin et al. 1996) that large planets observed close to their parents are initially located at larger 
Table 1. Stellar basic parameters, the companion information, kinematics and relative abundances for HD 190228.

\begin{tabular}{|c|c|c|c|c|c|c|c|c|c|c|c|c|c|c|c|c|}
\hline $\begin{array}{l}T_{\text {eff }} \\
(\mathrm{K})\end{array}$ & $\begin{array}{l}\log g \\
(\mathrm{cgs})\end{array}$ & {$[\mathrm{Fe} \mathrm{I} / \mathrm{H}]$} & $\begin{array}{c}\xi_{\mathrm{t}} \\
\left(\mathrm{km} \mathrm{s}^{-1}\right)\end{array}$ & $M_{V}$ & $\begin{array}{c}M_{1} \\
\left(M_{\odot}\right)\end{array}$ & $\begin{array}{c}\text { Age } \\
(\mathrm{Gyr})\end{array}$ & $\begin{array}{c}M_{2} \\
\left(M_{\mathrm{J}}\right)\end{array}$ & $\begin{array}{c}a \\
(\mathrm{AU})\end{array}$ & $\begin{array}{c}P \\
(\mathrm{yr})\end{array}$ & $e$ & $\underline{U_{\mathrm{LSR}}}$ & $\frac{V_{\mathrm{LSR}}}{\left(\mathrm{km} \mathrm{s}^{-1}\right)}$ & $W_{\mathrm{LSR}}$ & $R_{\max }$ & $\begin{array}{l}R_{\min } \\
(\mathrm{kpc})\end{array}$ & $Z_{\max }$ \\
\hline 5180 & 3.7 & -0.40 & 1.3 & 3.35 & 1.33 & 2.95 & 5.0 & 2.31 & 3.09 & 0.43 & 9.6 & -41.2 & -28.7 & 8.53 & 5.85 & 0.37 \\
\hline$[\mathrm{Fe}$ II $/ \mathrm{H}]$ & {$[\mathrm{C} / \mathrm{Fe}]$} & {$[\mathrm{O} / \mathrm{Fe}]$} & {$[\mathrm{Na} / \mathrm{Fe}]$} & {$[\mathrm{Mg} / \mathrm{Fe}]$} & {$[\mathrm{Al} / \mathrm{Fe}]$} & {$[\mathrm{Si} / \mathrm{Fe}$} & {$[\mathrm{S} / \mathrm{Fe}$} & {$[\mathrm{K} / \mathrm{Fe}]$} & {$[\mathrm{Ca} / \mathrm{Fe}]$} & {$[\mathrm{Sc} / \mathrm{Fe}]$} & {$[\mathrm{Ti} / \mathrm{Fe}]$} & {$[\mathrm{V} / \mathrm{Fe}]$} & {$[\mathrm{Cr} / \mathrm{Fe}]$} & $\mathrm{Mn} / \mathrm{Fe}]$ & {$[\mathrm{Ni} / \mathrm{Fe}]$} & {$[\mathrm{Ba} / \mathrm{Fe}]$} \\
\hline-0.35 & 0.01 & 0.08 & 0.18 & 0.14 & 0.16 & 0.12 & 0.20 & -0.05 & 0.04 & 0.11 & 0.01 & -0.03 & 0.01 & 0.05 & 0.03 & -0.02 \\
\hline
\end{tabular}

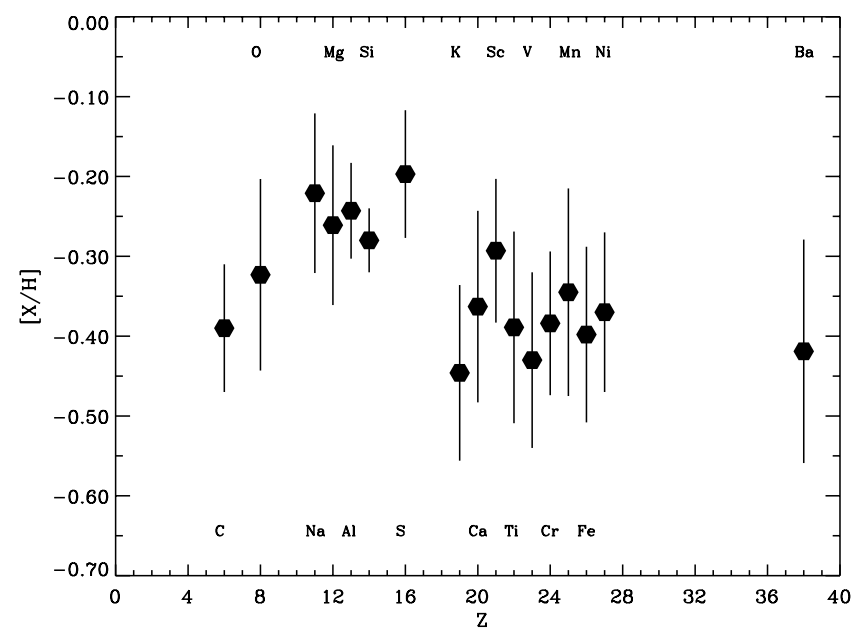

Fig. 1. Elemental abundances relative to the sun with error bars overplotted.

distances (i.e. beyond $4 \mathrm{AU}$ ), but migrate inwards by the help of drag and/or tidal forces. During the migration, parent stars might accret the fractionated material, which is depleted in $\mathrm{H}$ and $\mathrm{He}$, from the planetary disks. The deposit of a substantial amount of $\mathrm{H}$ - and He-deficit material in the envelope could enhance the surface metallicity of the parent star. Consistent with this hypothesis, $[\mathrm{Fe} / \mathrm{H}]$ excess for massive parent stars are found by Gonzalez et al. (2001) and the positive slopes of $[\mathrm{X} / \mathrm{H}]$ versus condensation temperature ( $\left.T_{\text {cond }}\right)$ of the element for six stars with the mean mass of $1.23 M_{\odot}$ are witnessed by Smith et al. (2001). With a mass of $1.33 M_{\odot}$, both effects should be easily discovered for HD 190228 if the formation of the planet does affect its composition. Unfortunately, the former has been denied by its low metallicity. The second effect is also marginal as shown in Fig. 2, where the refractory elements (Fe, Cr, Mn, Ni), which are thought to be preferentially accreted by the parent star, have the same $[\mathrm{X} / \mathrm{H}]$ as those of violate elements $(\mathrm{C}, \mathrm{O})$. Moreover, elements with intermediate $T_{\text {cond }}$ (e.g. $\mathrm{S}$ and $\mathrm{Na}$ ) show even higher abundances than those with the highest $T_{\text {cond }}(\mathrm{Mg}$, $\mathrm{Si}, \mathrm{Sc}$ and $\mathrm{Al}$ ); this seems to support the suggestion of the initially low metallicity according to Smith et al. (2001), who found a decreasing slope of $[\mathrm{X} / \mathrm{H}]$ versus $T_{\text {cond }}$ as $[\mathrm{Fe} / \mathrm{H}]$ decreases. In short, we suggested that abundances of this star are not polluted by the presence of the planet.

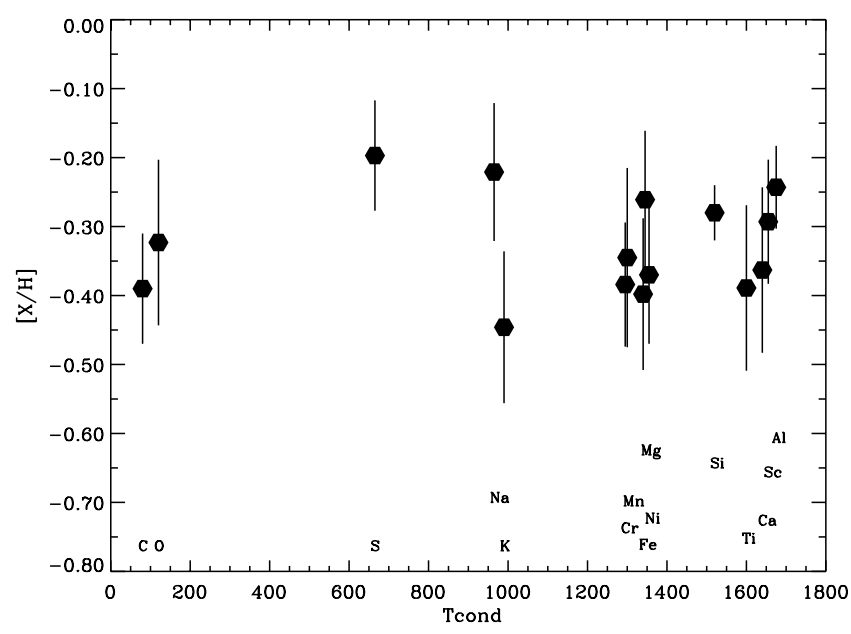

Fig. 2. $[\mathrm{X} / \mathrm{H}]$ vs. condensation temperature of the element (taken from Lodders \& Fegley 1998).

\subsection{Lithium abundance and stellar evolution}

It was suggested that stellar Li abundance might be enhanced as a result of the accretion of rocky material (Alexander 1967; Gonzalez 1998). The Li I $\lambda 670.8 \mathrm{~nm}$ of HD 190228 is very weak with $E W \lesssim 70$ pm, which corresponds to a $\mathrm{Li}$ abundance of $\lesssim 0.8$ dex. There are two ways to explain the low $\mathrm{Li}$ abundance for its high mass. On one hand, its Li could have been depleted by the unknown "Li-dip" mechanism as the mass and metallicity of HD 190228 follow the relation of the "Li-dip" stars found by Chen et al. (2001). On the other hand, from its position in the HR diagram, HD 190228 is a subgiant; this is also confirmed from its surface gravity. According to Sackmann et al. (1993), the outer convective zone of the sun could increase by $0.4 M_{\odot}$ at the end of subgiant stage. Based on this, one may expect a significant dilution in the composition of HD 190228 during the subgiant evolution. This suggestion is, however, challenged by the normal Li abundance observed in HD 38529 (Zhao et al. 2001), which is a massive planet-harboring subgiant with similar age. Moreover, another planet-harboring subgiant, HD 177830, together with HD 38529, are all more metal-rich than 0.2 dex. Finally, an increase in mass of the outer convective envelope by $0.4 M_{\odot}$ during the subgiant evolution of the sun, actually, has no effect on abundances of heavy elements (Sackmann et al. 1993). Such an effect should be negligible for HD 190228 although we don't know how significant increase of its outer convective 
envelope is. This is consistent with observations that stellar surface composition of subgiants is similar as that of turnoff stars. All of these argue against the suggestion that the subgiant evolution of HD 190228 has significantly changed its initial composition. In addition, the possibility of engulfing nearby planets during the expansion of this subgiant can be excluded by the lack of evidence for the enhanced metallicity and the positive slope of $[\mathrm{X} / \mathrm{H}]$ vs. $T_{\text {cond }}$.

\section{Possible solution}

Although we can accept that the initial metallicity as low as -0.4 for HD 190228 could still meet the condition of the planet formation, it is difficult to understand why the process of planet formation, which has enriched the metallicities of the majority of planet host stars, does not happen on this star.

One may argue that a large semi-major axis (2.31 AU) for HD 190228 would indicate only a slight migration of the planet from primordial location and the corresponding long period could make the migration proceed slowly. Thus, the metallicity could have not been significantly enhanced by the process. This suggestion is challenged by the results of other four stars (HD 22049, HD 95128, HD 145675 and HD 10687) with semi-major axes beyond $2 \mathrm{AU}$ and period longer than 1000 days; they are found to be enhanced in metallicity by $0.2-0.4$ dex as compared to their ages and mean galactocentric distances. If HD 190228 is surrounded by a planet, this amount would be more significant because of its massive companion.

It seems that a scenario other than the planet formation is required. Actually, there is a reasonable explanation for the low metallicity of HD 190228. Based on the high companion mass of $5.0 M_{\mathrm{J}}$, which is lower limit of the real value because of the unknown $\sin i$, we suspect that the companion is a brown dwarf instead of a planet. We have found that the photometrically-derived metallicities of seven stars accompanied by brown dwarfs (i.e. mass above $10 M_{\mathrm{J}}$ ), namely HD 18445, HD 29587, HD 89707, HD 98230, HD 110833, HD 112758 and HD 114762, span from -1.0 to -0.2 , and only HD 140913 has $[\mathrm{Fe} / \mathrm{H}]=0.0$. The formation of brown dwarfs may not be related with the parent star, and thus the metallicity distribution is normal. In particular, it is not necessary that the parent star is metal rich.

\section{Concluding remarks}

We have derived the atmospheric parameters, mass, age and kinematics for HD 190228 as well as the detailed abundances from high quality spectra. In contrast to the majority of extrasolar planet candidates, this star is very metal-poor relative to the average of planet host stars, and the abundance pattern is not special under the context of the galactic evolution of the disk. We suggest that its low metallicity is initial since the formation of the planet and the subgiant evolution do not change its composition.
With the lacks of $[\mathrm{Fe} / \mathrm{H}]$ excess and a positive slope of $[\mathrm{X} / \mathrm{H}]$ vs. $T_{\text {cond }}$, the hypothesis of the polluted material by the presence of the planet is denied as a possible process acting on HD 190228. Combining this with the high $M_{2}$, we suspect that HD 190228 is accompanied by a brown dwarf, not a planet. This suggestion is supported by the normal abundance pattern and the peculiar orbit parameters - long period, large semi-major axis and high eccentricity of this star; these are not normal for a planet companion especially when they are compared with the solar system.

Acknowledgements. We are grateful to Dr. Y. Takeda for providing the non-LTE grids, Dr. H. Abt and the referee Dr. G. Gonzalez for useful comments. This work is supported by NKBRSF G1999075406 and the National Natural Science Foundation of China under grant 19725312.

\section{References}

Alexander, B. 1967, Observatory, 87, 238

Allen, C., Schuste, W. J., \& Poveda, A. 1991, A\&A, 244, 280

Alonso, A., Arribas, S., \& Martínez-Roger, C. 1996, A\&A, 313, 873

Chen, Y. Q., Nissen, P. E., Zhao, G., Zhang, H. W., \& Benoni, T. 2000, A\&AS, 141, 491

Chen, Y. Q., Nissen, P. E., Benoni, T., \& Zhao, G. 2001, A\&A, in press

Cui, C. Z., Zhao, G., Zhao, Y. H., \& Shi, J. R. 2000, Science in China A, 30, 953

ESA 1997, The Hipparcos and Tycho Catalogues, ESA SP-1200

Edvardsson, B., Andersen, J., Gustafsson, B., et al. 1993, A\&A, 275,101

Gustafsson, B., Harlsson, T., Olsson, E., Edvardsson, B., \& Ryde, N. 1999, A\&A, 342, 426

Kurucz, R. L. 1993, CD-ROM 13, Smithsonian Astrophys. Obs.

Gonzalez, G. 1997, MNRAS, 285, 403

Gonzalez, G. 1998, A\&A, 334, 221

Gonzalez, G. 1999, MNRAS, 308, 447

Gonzalez, G., Laws, C., Tyagi, S., \& Reddy, B. E. 2001, AJ, 121,432

Marcy, G. W., Cochran, W. D., \& Mayor, M. 1999, in Protostars and Planets IV, ed V. Mannings, A. Boss, \& S. R. Russel (University Arizona Press, Tucson)

Johnson, D. R. H., \& Soderblom, D. R. 1987, AJ, 93, 864

Lin, D. N. C., Bodenheimer, P., \& Richardson, D. C. 1996, Nature, 380, 606

Lodders, K., \& Fegley, B. 1998, in The Planetary Scientist's Companion (Oxford University Press, New York)

Olsen, E. H. 1993, A\&A, 102, 89

Sackmann, I.-J., Boothroyd, A. I., \& Kraemer, K. E. 1993, ApJ, 418, 457

Shi, J. R., Zhao, G., \& Chen, Y. Q. 2001, A\&A, in preparation Smith, V. V., Cunha, K., \& Lazzaro, D. 2001, AJ, in press

Schuster, W. J., \& Nissen, P. E. 1989, A\&A, 221, 65

Thorén, P., \& Feltzing, S. 2000, A\&A, 363, 692

VandenBerg, D. A., Swenson, F. J., Rogers, F. J., Iglesias, C. A., \& Alexander, D. R. 2000, ApJ, 532, 430

Zhao, G., Chen, Y. Q., Qiu, H. M., \& Li, Z. W. 2001, submitted to Chinese Journal of Astronomy and Astrophysics 